TITRE: QUELS «SIGNES 》 DANS QUELS « LANGAGES 》 POUR QUI VEUT INTERROGER LA DIMENSION ESTHÉTIQUE D'UNE EXPÉRIENCE ? FORMES PROFANES, FORMES EXPERTES.

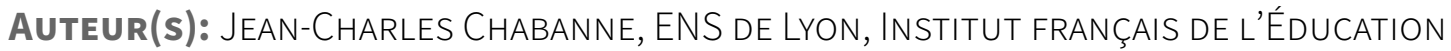

Publication: Discours, usAges, tRACES DE L'EXPÉRIENCE ESTHÉTIQUE EN CONTEXTE SCOLAIRE. PERSPECTIVES CROISÉES.

Directeurs: Marie-Christine Beaudry, Sylvain Brehm et JeAn-François Boutin

PAGES: $85-98$

URI: HTTP://HDL.HANDLE.NET/11143/14642

DOI: HTTPS://DOI.ORG/10.17118/11143/14642 


\section{Quels « signes » dans quels « langages » pour qui veut interroger la dimension esthétique d'une expérience ? Formes profanes, formes expertes.}

Jean-Charles Chabanne, ENS de Lyon, Institut français de l'Éducation, professeur titulaire.

Résumé : Cette contribution présente une réflexion à partir des trois questions posées lors de la journée : Comment définir l'expérience esthétique ? Peut-on l'évaluer? Quelle compétence professionnelle est-elle sollicitée ? Les réponses sont données dans le cadre d'un projet de recherche-intervention-formation d'enseignants de classes préélémentaires dont la mission est justement d'initier à une éducation artistique et culturelle, qui comporte une dimension d'éducation esthétique (le « sensible »).

Mots-clés : Éducation esthétique, éducation artistique et culturelle, gestes professionnels, expérience esthétique.

Aesthetic education - Art education - Professional competence - Aesthetic experience. 


\section{Introduction}

La journée d'études organisée le 17 mai 2017 à Montréal nous invitait à répondre à trois questions, qui ne sont pas des plus faciles: Comment définissez-vous «expérience esthétique »? Doit-on, peut-on "évaluer » l'expérience esthétique - et comment? Quel doit être, quel peut être le travail des éducateurs à l'échelle fine de leurs savoir-faire professionnels? Si la définition de la dimension esthétique de l'expérience est très vaste, elle se démultiplie à deux niveaux: d'abord, quand on l'aborde sous l'angle proposé, qui est celui de son «éducation » («transmission » ?« enseignement »? « initiation »?, etc., chacun de ces termes étant lui-même à questionner) ; ensuite, quand on l'aborde sous l'angle des « savoir-faire professionnels » qui seraient sollicités par cette opération d'éducation à l'expérience esthétique, que nous préférons reformuler en « dimension (ou qualité) esthétique de l'expérience».

La question ainsi posée est vaste. Nous l'abordons pour notre part dans un contexte applicatif, d'une part, et d'un point de vue restreint à l'étude des pratiques sémiotiques des acteurs.

\section{Contexte applicatif}

Le contexte « applicatif » est celui de la formation d'enseignants non spécialistes d'une des disciplines artistiques qu'il doit cependant, au terme de son contrat professionnel, « enseigner » ou simplement « faire pratiquer » aux élèves. Avec des enseignants en formation, qu'ils soient débutants ou même expérimentés, nous butons un moment ou un autre sur la nécessité d'évaluer la qualité esthétique ou artistique (nous y reviendrons) d'une activité proposée aux élèves, ou de telle ou telle production. Cette évaluation n'est pas réduite à la notation, mais dans le sens très pratique et très usuel de «savoir où l'on en est », de « savoir ce que ça vaut » en regard d'attendus prescrits, et de « savoir ce qu'on va faire » à un moment donné. Par exemple : qu'est-ce qui fait de ce moment de dessin un moment intéressant du point de vue d'une éducation à l'art? Ou encore : qu'est-ce qui fait la différence entre une séance de lecture ou d'écriture « ordinaire », et une séance de lecture ou d'écriture « littéraire » : est-ce que cette distinction même a un sens ? Ou encore : comment conduire le débat qui s'engage sur l'intérêt relatif de deux productions du même élève (plastique, textuelle, gestuelle...) ? Nous ne considérons pas ces questions tranchées d'avance par quelque cadre théorique robuste et préexistant : ce sont, très naïvement, des questions qui font la complexité du travail ordinaire de l'enseignant.

\section{Pratiques sémiotiques des acteurs}

Venons-en à notre second angle d'attaque. Pour aborder les situations où « quelque chose de l'art/de l'esthétique » serait en jeu, nous choisissons une approche sémiotique, à savoir : nous observons les jeux de langage (Wittgenstein, 1953)»edition»:»Trad. fr. 2005»,»archive_location»:»perso»,»event-place»:»Paris»,»call-number»:»30/08/2005»,»author»:[\{«family»:»Wittgenstein»,»given»:»Ludwig»\}],»issued»:\{«date-parts»:[[«1953»] ]\}\}] ,»Schema»:»https://github.com/citation-style-language/schema/raw/master/csl-citation.json»\} , les circulations de signes (verbaux, non verbaux...) qui leur sont associés. Et nous considérons ces pratiques sémiotiques comme intrinsèquement liées à ces situations, et donc à leur éventuelle qualité esthétique. En particulier, nous nous intéressons justement aux discours d'évaluation de cette dimension que les acteurs produisent d'eux-mêmes. Nous considérons ainsi que les situations qui sont pertinentes relèvent avant tout du point de vue des acteurs engagés, dès lors qu'ils expriment, sous une forme ou une autre, que « quelque chose d'une expérience esthétique » est en jeu et qu'ils s'engagent dans une négociation à propos de cet en-jeu. 
Ce choix de point de vue doit aussi se comprendre à partir du point d'où je parle, comme on disait dans ma jeunesse. Mon domaine de formation académique est celui des études littéraires, que j'aborde comme un des domaines des arts, et non isolé, comme y invitent les textes officiels français qui définissent l'éducation artistique et culturelle [ÉAC] (MEN, 2015). Parmi les objets traditionnels des études littéraires, il y a justement un genre constitué de toutes sortes d'écrits qui portent justement sur l'évaluation de la dimension esthétique de certaines expériences (artistiques souvent, mais pas uniquement) : il s'agit du genre des écrits sur l'art, genre dont les spécialistes eux-mêmes soulignent l'extrême hétérogénéité (Vouilloux, 2005 ; Vaugeois, 2005). Ce champ voisine avec des pratiques sociales comme la critique (artistique), et de proche en proche avec la plupart des sciences de l'art, sémiologie, esthétique, histoire, sociologie de l'art (dont un des thèmes est justement la déconstruction des présupposés des discours sur la valeur de l'art, sur les effets de légitimation et de sacralisation à l'œuvre : Bourdieu, 1998 ; Heinich, Schaeffer et Talon-Hugon, 2014 ; Lahire, 2015).

\section{Approche sémiotique des pratiques scolaires}

Notre propos est d'éclairer les pratiques scolaires de la parole sur l'art par la problématique des écrits sur l'art, y compris dans l'effet de mis en abîme que constitue le fait que la parole sur l'art peut être considérée comme un art, en quelque sorte au second degré. Et cette pratique sociale « de référence » éclaire pour moi à la fois le grand intérêt et la grande difficulté réelle de nombreuses tâches considérées comme des allant-de-soi dans l'enseignement culturel et artistique, à savoir les consignes qui invitent les élèves à « parler de » ce que représente une œuvre, ce qu'elle veut dire pour eux, ce qu'ils « ressentent », etc.

Car les écrits sur l'art (qu'ils soient légitimés comme les écrits d'écrivains ou d'artistes sur d'autres artistes; ou moins légitimés comme les écrits journalistiques; et encore moins légitimés, les écrits et paroles de profanes, d'apprentis ou d'élèves), sont en lien direct avec la question posée : il s'agit bien pour ce type de discours de tenter d'exprimer la qualité esthétique de certaines expériences, voire d'argumenter et de théoriser à son propos. Et cette question est centrale pour ceux qui ont charge d'une « éducation à » des conduites esthétiques (apprécier, ressentir, interpréter...), puisqu'ils doivent référer à la dimension esthétique de l'expérience pour en faire un objet d'étude - même si la « compétence esthétique » ne se construit pas par le truchement du langage, il faut a minima créer discursivement et sémiotiquement les conditions de son développement.

\section{Ma réponse à la question 1 : Définir la dimension esthétique de l'expérience}

Ce contexte une fois explicité, tentons de répondre à la première question posée : quelle définition donnons-nous à « expérience esthétique ». Le concept est tout à fait central dans toute la philosophie de l'art depuis Platon, et le chantier est loin d'être clos, car, comme le dit Chateau, « je crois que la baraque est hantée et que les questions qui l'habitent résistent à la radicalité de l'hygiène analytique» (1994, p. 145). Ce fantôme qui hante selon lui la philosophie de l'art, et plus généralement la réflexion esthétique, est justement celui de la définition, de l'identification et donc de l'évaluation de la qualité esthétique d'une expérience (voire d'un objet ou d'un phénomène). L'affirmation de Kant (1791), pour qui cette qualité peut être l'objet d'un jugement valide, est discutée autant par ceux qui veulent en montrer la nature essentiellement relationnelle et conventionnelle et que par les partisans d'une esthétique qui préserverait une part d'essentialisme².

1. C'est le postulat épistémologique de la philosophie analytique que Chateau questionne, celle de Dickie (1964), de Danto (1964), de Genette $(1994,1997)$, par exemple - et bien sûr celle de la sociologie de l'art.

2. Dewey (1934), Beardsley (1988), Pouivet (2010) et Réhault (2013), par exemple. 
Ces questions sont difficiles, et nous nous mettons dans les pas d'un guide savant comme Schaeffer (2015) pour les débrouiller. Schaeffer me semble avoir apporté ceci : il existe sans doute non pas une expérience esthétique perse, mais une dimension (qualité) esthétique de l'expérience, concept auquel nous donnons toute l'extension qu'en donne la philosophie pragmatiste avec Dewey. Schaeffer sollicite pour aborder cette dimension, entre autres, les apports des disciplines (neuro)cognitives. Il l'aborde donc, en quelque sorte, « de l'intérieur » du sujet, comme une variante particulière, mais une variante seulement, des processus de traitement perceptifscognitifs, qui serait spécifiée par des phénomènes d'intensification, de complexification, qui ne sont pas sans évoquer les propriétés que Dewey identifie comme celles d'un stimulus esthétique. Schaeffer lui-même évoque à plusieurs reprises la possibilité que le stimulus perceptif ne soit pas étranger à la mise en « mode esthétique » des processus de traitement, en particulier quand certains artefacts sont intentionnellement produits à cet effet (ou placés dans des contextes spécifiques : cette manière de présenter les choses conviendrait mieux aux sociologues et aux partisans d'une esthétique relationnelle et conventionnelle).

L'expérience esthétique est donc, selon Schaeffer, cognitive dans un sens riche, c'est-à-dire en-deçà des dualismes pensée-corps, ou concept-percept, ou percept-affect. Elle est caractérisée par une certaine qualité de l'attention, une mise en éveil, qui est susceptible d'être éduquée, ce qui nous intéresse. Schaeffer (2015) et Goodman (2005) parlent d'une densification attentionnelle, d'une saturation attentionnelle. Cette composante perceptive est inséparable d'une composante émotionnelle : une expérience est esthétique au sens où elle met en jeu, non pas précisément des émotions différenciées, que pourraient caractériser la psychologie des émotions (Cosnier, 1994 ; Damasio, 2010), mais une «valence », qui peut être positive ou négative. Soit une dimension hédoniste spécifique, le « plaisir esthétique » ou son inverse le déplaisir esthétique. On voit comment cette dimension affective peut se lier à une composante axiologique, relevant d'un jugement (et de débats) de valeur. Très concrètement, l'anthropologie des pratiques artistiques montre que des expériences de ce type, bien que dénuées en apparence d'un intérêt pratique immédiat, sont valorisées et recherchées, elles ont un prix parfois considérable.

\section{Définir la notion d'" expérience ", ou plutôt les notions que ce terme unique confond}

\section{L'expérience comme « rencontre », "événement »}

L'autre abîme conceptuel ouvert par les questions posées est la notion même d'expérience. Une tradition remontant à la phénoménologie distingue utilement deux concepts : Erlebnis/Erfahrung (Mayzaud, 2005). D’une part, Erlebnis, qui désigne l'expérience comme événement vécu dans sa semelfactivité (Jankélévitch, 1980), dans son unicité d'événement. C'est peut-être à cette singularité événementielle que réfère un terme qu'on voit revenir, de manière récurrente, dans les prescriptions définissant l'ÉAC : rencontre avec l'oeuvre/rencontrer une oeuvre, pour désigner ce qui est beaucoup plus qu'un événement isolé, mais se cristallise dans un moment, en général initial, qui peut être répété, voire décalé très loin dans le temps, quand se fait pleinement l'expérience esthétique d'une œuvre (qu'on regarde, qu'on écoute, etc.). L'écueil en ce point est d'aller directement à la sacralisation du "vécu » et de l' «instant magique ». On s'en tiendra à distance même si une grande partie des politiques de la culture se sont bâties sur l'idée que certains événements, des rencontres assez fortes avec des œuvres d'art, pouvaient servir de clef d'entrée dans les pratiques culturelles souhaitées. On trouve cela par exemple chez Malraux (1965), mais aussi, en didactique de la littérature, dans une certaine tradition pédagogique qui affirmai sa «foi dans la vertu des beaux textes » (le titre est de Clarac, 1963), donc la lecture seule devait emporter les élèves. Un tel duel eut lieu dans les années 90, quand Henri Mitterand (1992) croisait le fer 
avec les militants de Pratiques (Privat, 1995), mais les lames avaient aussi cliqueté entre Barthes et Raymond Picard (1965 ; voir Compagnon, 2011), ou entre Genette (1984) et Fumaroli (1984) à propos de « Comment lire la littérature».

Notons que les vents tournent, en didactique de la littérature comme ailleurs, et que revient sur le devant de la scène théorique la promotion d'approches empathiques et expérientielles ${ }^{3}$, voire «transitionnelles » de la littérature enseignée (au sens d'une culture des Erlebnissen).

Si l'Erlebnis est toute entière dans ces moments, dans ces événements, la question suivante est le passage d'une série d'événements aussi singuliers à la construction d'une éducation esthétique, ou encore d'une compétence esthétique que certains préfèrent appeler une sensibilité (Lemonchois, 2003), dans un contexte éducatif où ce qui est justement à construire est à la fois un sujet sensible et sa culture (Bildung). Le second concept d'Erfahrung désigne cette forme de connaissance résultant d'une élaboration des Erlebnissen en un répertoire de connaissances (sens 1) : le professeur de littérature, comme les autres enseignants en charge du projet de Parcours en ÉAC, ou encore les médiateurs au musée, ne sont pas seulement des organisateurs de happenings et de performances. Sans doute sont-ils en charge d'enrichir en priorité le répertoire d'expériences (esthétiques) des élèves, au titre d'autant d'événements singuliers et subjectifs de rencontre, si possible intéressantes à défaut d'être toujours plaisantes. La question professionnelle est donc bien celle-là : comment élaborer de l'expérience (volatile) en culture?

C'est ici qu'il faut éviter le passage trop rapide à l'abstraction pour aller voir de plus près, selon une méthode ethnologique, les pratiques qui accomplissent, dans des situations réelles, les opérations que nous venons d'évoquer. Ces pratiques sont pour partie sémiotiques. Nous observons que l'événement de la rencontre, même s'il est radicalement singulier et instantané, ne s'y réduit pas.

D’abord l'expérience esthétique peut s'inscrire dans une durée, une progressivité ou des moments disjoints. Arasse raconte comment, bien qu'il soit un spécialiste du domaine, son " expérience » d'un tableau aussi connu et commenté que La Joconde ne cesse pas de se renouveler ou de se développer tout au long de sa vie d'historien d'art. II raconte comment par exemple il redécouvre un certain détail du décor d'arrière fond à partir de quoi il ne reconstruit pas seulement des connaissances sur l'œuvre, sa fabrication, sa signification, mais comment il s'émerveille de cette découverte et de ce qu'elle renouvelle son appréhension sensible de l'œuvre (Arasse, 2006). C'est un exemple d'un écrit sur l'art, à finalité didactique (à l'origine, une émission de radio grand public), qui participe d'un projet global d'éducation, et c'est pourquoi le travail textuel d'Arasse retient notre attention. Il illustre le rôle des signes dans un travail de restitution d'une expérience à dimension esthétique, en vue de faire partager, non seulement les connaissances qui son celles de l'expert, mais, peut-être même avant tout, sa propre compétence sensible, sa propre sensibilité esthétique. Ses Histoires de peintures sont avant tout des récits d'événements, des histoires de rencontres avec des œuvres, le récit de l'élaboration de singularités en contenus communicables.

Arasse retrouve ici, très consciemment, le modèle des Salons de Diderot (1968). Avec les moyens qui sont les siens à l'époque, Diderot en effet ne cherche pas d'abord à diffuser des connaissances, voire simplement des informations factuelles sur les fameux Salons de Paris, dont le monde cultivé de l'époque, jusqu'à Moscou, est curieux. Il témoigne d'une expérience, sur un ton apparemment très personnel, voire " débraillé », comme si nous suivions devant les œuvres exposés son propre personnage du Neveu de Rameau, à la fois érudit, joueur,

3. Le mouvement, significativement lancé par un des théoriciens de référence du formalisme (Todorov, 2007), s'amplifie aujourd'hui (Petit, 2002 ; Citton, 2007 ; Vernay, 2013 ; Merlin-Kajman, 2016, etc.). 
et dilettante - mais si plaisant compagnon qu'on le suit, à la fois amusé et désapprobateur. La relation qui s'établit est d'expérience à expérience, de sensible à sensible. En cela, le travail sémiotique des Salons peut être une introduction à la problématique de la médiation et de l'éducation à la dimension esthétique de l'expérience.

On observe immédiatement que cette expérience n'est donc pas instantanée ni mécanique, mais aussi qu'elle n'est pas égocentrique et idiosyncrasique. Elle est sociale, et sans doute de bout en bout. L'expérience n'est pas seulement un phénomène interne et subjectif. II faut aussi l'aborder comme un phénomène social et sémiotique.

\section{Réponse à la question 2 : l'évaluation comme « traduction »}

\section{Évaluation des traces sémiotiques}

Certaines pratiques sociales sont spécifiquement organisées autour de telles expériences (production, consommation, évaluation, interprétation, éducation, etc.). Le « plaisir esthétique » est l'objet d'une production sociale, et son évaluation est l'objet d'interactions inégalement formalisées et institutionnalisées. Évaluation, traduction, interprétation, commentaire... ou encore éducation-transmission : l'expérience esthétique est l'objet de transactions multiples qui se manifestent par des traces sémiotiques, aux différents moments de son parcours.

On peut en lister quelques-unes.

- En amont : négociations, préparations, définitions d'intentions, etc., qui correspondent pour nous, éducateurs, à ce qui constitue le seuil des séances ou des moments organisés par les éducateurs en ÉAC.

- In situ et ex temporane, la « leçon d'expérience esthétique » est celle qu'accomplissent en particulier les professionnels de la médiation dans les musées et les expositions : par la verbalisation, mais aussi par d'autres modalités sémiotiques moins évidentes à identifier, par exemple comme la communication non verbale, le langage corporel, le discours métaphorique, voire le truchement d'autres œuvres jouant le rôle d'interprétant dans le processus (au sens de Peirce, 1978).

- En aval de l'expérience, à distance plus ou moins grande, on peut observer une « trainée interprétative » constituée de négociations, de questionnements, de traductions, d'argumentations, de verbalisations d'affects (euphorique/dysphoriques «les tableaux de Bacon me laissent toujours une impression désagréable...»).

Nous avons proposé, dans un travail précédent (Chabanne, 2017), d'aborder cette prolifération sémiotique autour de la dimension esthétique à la lumière de la théorie de la traduction, selon les différentes catégories analysées par Eco (2006), y compris les procédés de transmodalisations (soit le recours à des langages autres que la langue naturelle).

Ce sont ces traces qui constituent les matériaux pour le chercheur mais, avant tout, elles sont aussi des indices pour les acteurs : les visiteurs du musée écoutant la médiatrice et regardant l'œuvre ; les auditeurs d'une émission de commentaire musical ; les élèves d'une master class; les membres d'un ciné-club à la fin de la projection ; les internautes regardant un clip où un amateur explique ce qu'il a aimé ou pas aimé de l'actualité du cinéma ou des jeux vidéo... Tous font usage spécifique de signes pour parler de la dimension esthétique et la constituer sinon en savoir, du moins en en-jeu de l'interaction. 
Sauf dans des cas très particuliers, qui à ce titre devraient faire l'objet d'études plus précises, comme les émissions de critique en arts visuels à la radio, les phénomènes que nous évoquons sont très largement plurisémiotiques ou, comme on le dit aujourd'hui par un calque de l'anglais, plurimodaux. Quelques exemples:

- $\quad$ du point de vue du didacticien, le milieu qu'on peut analyser dans une leçon de danse est irréductiblement hybride sémiotiquement : le corps, évidemment, y est un signe, d'où par exemple l'importance des miroirs qui tapissent au moins un des murs de la salle de répétition. Le corps du maitre, sans doute, qui parle abondamment, d'abord en dansant lui-même (souvent d'ailleurs de manière plus allusive que monstrative : le maitre danse moins et parfois moins bien que le danseur), mais aussi via le langage du corps : mimiques, postures, gestuelle... Ou encore le langage du paraverbal phonique (Kerbrat-Orrechioni, 1990), intonations, sons non phonémiques, esquisses chantées parfois, etc. En outre, le langage du danseur et de ses partenaires participe aussi du dispositif sémiotique : ainsi, c'est aussi en dansant que le danseur commente et catégorise les contenus de pensée qu'il cherche à faire passer, dont la complexité ou la subtilité par ailleurs épuise les ressources verbales : comment décrire par exemple, au-delà du glossaire technique (tendu, gainé, relâché, souple, élégant...) et des métaphores improvisées (Parsons, 2010), les effets précis de qualité du mouvement ou d'états de corps éprouvés par le danseur ou visés par le chorégraphe : il ne reste plus que le geste dansé pour parler du geste dansé.

- Une analyse comparable peut être faite lors d'une séance d'arts plastiques. Le milieu est constitué de plusieurs modalités, dont la moins importante n'est pas le matériel, le support, et la trace de l'outil : mais il faut observer avec soin comment le commentaire de l'enseignant focalise l'attention sur certaines dimensions du geste technique ou de la trace produite; il en accompagne le tracé parfois en temps réel, comme s'il « prenait la man ». Mais pour l'apprenti-plasticien, sa propre trace est signe pour l'apprentissage d'où par exemple l'importance de regarder ce qu'on produit, geste d'artiste par excellence (se reculer, comparer des esquisses...). Les différents états d'un même tableau, ou les pages du carnet de croquis, sont autant d'états intermédiaires du projet, mais aussi des signes avec lesquels il se construit.

- Enfin, dans l'histoire de l'art, les exemples sont nombreux de ces dialogues inter-arts (Vouilloux, 1997) où un artiste utilise le langage artistique qu'il maitrise pour en quelque sorte « trouver une langue » avec laquelle il commente une autre œuvre, ou même cherche un langage pour dire la dimension esthétique de l'expérience. Nous avons évoqué les écrits sur l'art avec Diderot, mais on pourrait donner des exemples de musiciens composant à propos de, en réaction à, des plasticiens; des plasticiens réagissant à des œuvres musicales (Bosseur, 1998); des danseurs nourris de mais aussi commentant des textes de fiction voire de théorie (Macel et Lavigne, 2011), et toutes sortes de combinaisons.

\section{Des pratiques savantes ou des pratiques profanes}

Ce qui nous intéresse ici est que ces pratiques savantes, légitimées, devraient nous aider à éclairer des pratiques plus modestes, mais qui importent en éducation artistique et culturelle car elles sont précisément les formes sémiotiques d'une éducation au sensible. Prenons l'exemple de cette tradition scolaire du cahier de poésies illustré, qui peut apparaitre comme une pratique charmante et désuète : sur la page de droite, soigneusement calligraphié, le poème de Maurice Fombeure ou de Jean-Pierre Siméon; sur la page de droit, un dessin. On voit bien tout l'intérêt de redonner un sens didactique à l'activité perçue comme routinière du travail de l'illustrateur, en le confrontant à des pratiques sociales de référence qui en éclairent les enjeux esthétiques autant qu'anthropologique. L'illustrateur est-il un traducteur-imitateur? Ou un interprète (au sens musical du terme), à la manière de Doré illustrant Rabelais ? Ou encore un interlocuteur, co-produisant avec l'écrivain le livre-objet? Et de tous ces rôles, quels sont ceux auxquels sont conviés les élèves : on voit bien que la place de 
l'esthétique dépend ici très clairement d'un contrat didactique, ou plus largement du sens donné à la tâche : quelle est la charge artistique de l'activité proposée ? On voit ici que la réponse relève d'un art de faire professionnel.

\section{Ma réponse à la question 3 : Quel art de faire professionnel ?}

Certaines des situations où " quelque chose de l'art est en-jeu » sont asymétriques : quelqu'un est là pour apprendre (découvrir, s'informer, être guidé...) de quelqu'un d'autre. Cette relation peut être très informelle, comme dans la conversation d'amis qui racontent leurs récentes sorties au cinéma ou dans une salle de concert. Elle peut être très formalisée, dans l'enseignement de haut niveau par exemple, en école d'art, en académie, en master class.

\section{Description d'une situation exemplaire}

Pour en rester à l'éducation à la dimension esthétique de l'expérience, nous avions proposé de l'illustrer à partir de l'analyse d'une situation à valeur exemplaire. Il s'agissait d'une situation d'éducation culturelle à destination d'enfants de Grande section (5-6 ans), qui visitent à Montpellier une exposition de sculptures un peu particulière, parce qu'elle a justement très explicitement une visée didactique explicite. Il s'agit de l'exposition L'Art et la matière, une Galerie de sculptures à toucher (depuis janvier 2017). L'exposition se présente dans une salle dont l'aménagement scénographique permet à des visiteurs de tous âges d'avoir à portée de la main des copies d'œuvres, la plupart au format original, de dimensions variées : statues en pied, groupes animaliers, bas-relief, statue équestre en réduction, etc. ${ }^{4}$. Le public est accueilli par un panneau d'initiation à l'exploration tactile (boites à toucher, échantillons de matériaux,...). Un autre espace expose quelques outils de modelage, l'éventail des techniques de la sculpture et un film sur le moulage au bronze (Chabanne et al., 2017).

Synopsis. La séance étudiée dure environ 45 minutes. Elle comporte trois phases: dans la première, les élèves, en petits groupes, font un premier parcours de l'exposition, les yeux bandés, aidés par les accompagnateurs qui les amènent devant les œuvres à toucher. À tâtons, ils parcourent les œuvres qui, dans la plupart des cas, sont à leur hauteur soit posées sur le sol, soit accessibles par des quelques marches. Dans un second temps, regroupés à l'écart, ils ont pour consigne de dessiner « ce qu'ils ont touché ». Dans la troisième phase, ils reviennent dans la salle, et essayent de retrouver ce qu'ils ont deviné par le toucher et échangent entre eux et avec l'accompagnateur.

\section{Analyse de la situation}

Nous avions trois questions pour l'analyse. La première était d'abord exploratoire et descriptive, à partir de cette étude de cas dans un dispositif muséal peu fréquent : qu'est-ce qui se passe ? La seconde portait sur les « contenus »: Qu'est-ce qui s'enseigne, s'apprend, se développe ou simplement est en-jeu dans cette situation, considérée a priori comme éducative, en lien avec les problématiques de la sculpture et de son appréhension par un regardeur-toucheur? À cette question est liée une sous-question méthodologique : comment les acteurs

4. Le Jacques Coeur d'Antoine-Augustin Préault (1873, h. 245 cm); La Frileuse (1783, h. 145 cm), L'été (1785, h. 155 cm), Voltaire (178090, h. $145 \mathrm{~cm})$, de Jean-Antoine Houdon; Tête de l'éloquence, de Bourdelle (1923, h. $49 \mathrm{~cm})$; L'enfant à l'oie, copie d'une statue du lle s. (h. $92 \mathrm{~cm})$; L'Amour au papillon, de Antoine-Denis Chaudet $(1817$, h. $77 \mathrm{~cm})$; Statue équestre de Bartolomeo Colleoni, de Joseph Marius Ramus (1855, h. 65 cm), d'après Andrea del Verrocchio ; La Vierge et l'enfant entourés par quatre anges (dite aussi la Madone d'Auvillers), de Agostino di DUCCIO (1469, bas-relief, h. 81 cm) ; Jaguar dévorant un lièvre, de Antoine Louis Barye (ap. 1850, h. 41 cm). (Sobczak, 2016, pp. 19-20) 
identifient-ils ces en-jeux à partir de traces observables, actions motrices, productions verbales, productions graphiques... ? Enfin, le groupe de recherche-intervention qui menait l'enquête cherchait à savoir quels éléments de compétence professionnelle, quels gestes professionnels pouvaient être mis en évidence dans cette situation. Sur ce point, l'observation laissait voir que les mêmes enfants, au même moment du parcours, se comportait très différemment selon qui était l'accompagnateur, parent, médiateur, enseignant.

Ces questions ne sont pas seulement théoriques. Pour le professionnel de l'enseignement ou de la médiation, ce sont les questions même de l'évaluation de la dimension esthétique de l'expérience, qui est l'objectif éducatifformulé par tous les acteurs, quel que soit leur profession. Il s'agit bien d'apprendre, très globalement, aux enfants à vivre ce type d'expérience vers laquelle converge tout le dispositif muséal : apprendre, donc, à être ce visiteur de musée, et plus largement, à long terme, à devenir cet amateur éclairé qui est l'objectif final du projet ÉAC (MEN, 2015).

Le moment observé en gros plan dure 2 min. 20 sec. Une dizaine d'élèves se regroupent autour d'une reproduction en modèle réduit (h. $65 \mathrm{~cm}$ ) de la Statue équestre de Bartolomeo Colleoni, de Joseph Marius Ramus, reproduction récente (1855) d'une statue d'Andrea del Verrocchio. C'est l'enseignant qui mène l'échange. Une des élèves du groupe parle plus, mais les autres suivent son commentaire en allant toucher les parties qu'elle désigne et en prenant la parole. Les élèves ont rapidement reconnu un cavalier et son cheval, mais s'arrêtent sur l'arme qu'il porte, et aussi sur des détails très fins : les jambes, les bottes, des éléments en relief que la perception par le toucher a fait saillir ; le détail du casque, sa visière, et le couvre-nuque, sur lequel ils reviennent à plusieurs reprises. Finalement, une comparaison va éclairer les explorateurs (nombreux acquiescements, reprise de la description) : le cavalier apparait équipé « comme les gendarmes en Algérie » : jambières, bouclier, et surtout casque.

Plusieurs questions sont ouvertes à partir d'ici : lorsqu'il revient sur ce moment filmé, l'enseignant se montre très précis dans ses propres analyses et compare les conduites des élèves avec ce qu'il sait d'eux en classe. Il constate la précision des détails fixés une première fois dans la phase de tâtonnement : sans doute, le format de l'objet, plus accessible par exemple que La Frileuse, presque en taille réelle, donne aux élèves un terrain sensible plus facilement lisible. La phase de dessin a permis de montrer que de nombreux détails avaient été perçus. Mais ce que les échanges font ressortir, c'est l'importance pour les élèves de traiter la statue comme porteuse de significations, qu'ils finissent pas arrêter en repérant les marques de sa fonction guerrière : elle fait peur, par l'exhibition selon eux de ses armes et de ses protections - la comparaison peut être anachronique, mais il faut sans doute la lire comme la solution à un problème sémiotique : quelque chose est perçu (menace, puissance...) de ce que signifie la statue, et l'historien de l'art est sollicité pour rendre compte de l'intention et de l'usage de l'œuvre originale. Mais sans doute ne sommes-nous pas très loin de ce que les élèves ont repéré.

\section{Conclusion : de la curiosité à la méthode}

La méthode appliquée ici se proposer de mettre en parallèle ces pratiques scolaires très humbles, ordinaires et peu saillantes, à des pratiques sociales plus visibles et plus légitimes : qui, dans les mondes de l'art, agit comme ces élèves et dans quelles intentions? Toucher la sculpture est un privilège : c'est celui de l'artiste, du fondeur, de l'historien d'art, du restaurateur..., disions-nous avec les concepteurs de la Galerie, le privilège ultime du propriétaire amateur. Toucher pour apprendre à voir? La question reste ouverte (Lemoine et Simier, 2016). Mais avant tout il s'agit ici très directement d'une formation au sensible que la Galerie met à disposition de ses visiteurs : un retour au sens matériel du mot "esthétique », ce qui se touche, mais aussi ce qui touche. Dans cette expérience, les élèves touchent mais aussi sont touchés par l'œuvre, qui a minima les intrigue, les 
questionne, et finalement, s'inscrit dans une narration où les souvenirs personnels viennent donner sens à une figuration venant de la Renaissance. Le Cavalier Colleoni impressionne ; il saisit dans ses formes la figure du Condottiere, à la fois effrayante et fascinante. II reste à apprendre ensuite ce que cela signifie à l'époque où l'original est exposé, et aussi pourquoi l'œuvre est reprise à l'époque romantique. Ceci est affaire d'historien d'art : ce que poursuit l'ÉAC se situe encore en-deçà : il s'agit d'initier à la perception sensible du signe artistique. L'approche savante viendra ensuite.

Enfin, ce que montre la très courte séance est un savoir-faire professionnel que commentent les acteurs. Une triple expertise : Lire les signes pour observer et comprendre la situation, savoir regarder les élèves finement. Produire des signes pour intervenir, à savoir guider, accompagner, étayer ce qui est en-jeu, et qui n'est pas encore un discours (celui du sémiologue, de l'historien de l'art) mais une disposition. II s'agit de produire des signes pour enseigner quelque chose est à « transmettre » et ne peut être re-construit par les élèves, cet objet labile que nous cherchions à saisir, la dimension esthétique comme expérience. 


\section{Bibliographie}

Arasse, D. (2006). Histoires de peintures. Paris : Gallimard.

Beardsley, M. (1988). L'expérience esthétique reconquise. Dans D. Lories (dir.), Philosophie analytique et esthétique (p. 143-157). Paris : Klincksieck.

Bourdieu, P. (1998). Les règles de l'art. Genèse et structure du champ littéraire. Paris : Le Seuil.

Bosseur, J.-Y. (1998). Musique et arts plastiques: interactions au XXe siècle. Paris : Minerve.

Chabanne, J.-C. (2017). L'espace interdisciplinaire entre la discipline « français » et l'Éducation artistique et culturelle. Recherches, Interdisciplinarités, (67), sous presse.

Chabanne, J.-C., Penancier, M.-Ė., Arbouet, F., Bertrand, D., Bidard, M.-D., Gaquerel, M., Savaldor, C. (2017). Autour de la sculpture et du tactile : jeux de langage(s), conduites d'élèves et arts de faire professionnels. Pratiques, 173-174, sous presse.

Chateau, D. (1994). La question de la question de l'art. Vincennes : Presses universitaires de Vincennes.

Citton, P. (2007). Lire, interpréter, actualiser. Pourquoi les études littéraires ? Paris : Amsterdam.

Clarac, P. (1963). La foi dans la vertu des beaux textes. Dans L'enseignement du français (p.144). Paris : PUF.

Compagnon, A. (2011). Barthes contre Picard. Cours en ligne du collège de France, 08 février. Repéré à http:// www.college-de-france.fr/site/antoine-compagnon/course-2011-02-08-16h30.htm

Cosnier, J. (1994). Psychologie des émotions et des sentiments. Paris : Retz.

Damasio, A. R. (2010). L'erreur de Descartes : la raison des émotions (traduit par M. Blanc). Paris : Odile Jacob.

Danto, A. C. (1964). The artworld. Journal of Philosophy, 571-584.

Dewey, J. (2010). L'art comme expérience (traduit par J. Cometti). Paris : Gallimard.

Dickie, G. (1964). Beardsley et le fantôme de l'expérience esthétique. Dans D. Lories (dir.), Philosophie analytique et esthétique (p. 135-142). Paris : Klincksieck.

Eco, U. (2006). Dire presque la même chose. Expériences de traduction. Paris : Grasset.

Fumaroli, M. (1984). Comment parler de la littérature ? Le Débat, (29), 140-143.

Genette, G. (1984). Comment parler de la littérature ? Le Débat, (29), 144-148.

Genette, G. (1994). L'oeuvre de l'art I: Immanence et transcendance. Paris : Le Seuil.

Genette, G. (1997). L'oeuvre de l'art, I/ : La Relation esthétique. Paris : Le Seuil.

Goodman, N. (2005). L'art en action. Dans J.-P. Cometti, J. Morizot et R. Pouivet (dir.), Esthétique contemporaine. Art, représentation et fiction (p.143-157). Paris : Vrin.

Heinich, N., Schaeffer, J.-M. et Talon-Hugon, C. (dir.). (2014). Par-delà le beau et le laid. Enquêtes sur les valeurs de l'art. Rennes: Presses universitaires de Rennes.

Jankélévitch, V. (1980). Le Je-ne-sais-quoi et le Presque-rien 1 : La manière et l'occasion. Paris : Le Seuil.

Kant, E. (1791). Critique de la faculté de juger (traduit par A. Renaut, 1995). Paris : Flammarion. 
Kerbrat-Orecchioni, C. (1990). Les interactions verbales (tome 1). Paris : Armand Colin.

Lahire, B. (2015). Ceci n'est pas qu'un tableau. Essai sur l'art, la domination, la magie et le sacré. Paris : La Découverte.

Lemoine, C. et Simier, A. (2016). La sculpture sur le bout des doigts. Retour sur l'élaboration d'une salle pédagogique et tactile au musée Bourdelle. Les cahiers de l'École du Louvre, (8). https://doi.org/10.4000/cel.341

Lemonchois, M. (2003). Pour une éducation esthétique. Discernement et formation de la sensibilité. Paris : L'Harmattan.

Macel, C. et Lavigne, E. (dir.). (2011). Danser sa vie. Art et danse de 1900 à nos jours. Paris : Editions du Centre Pompidou.

Malraux, A. (1965). Le musée imaginaire. Paris : Gallimard.

Mayzaud, Y. (2005). Historique et enjeu de la notion d'Erlebnis. Revue CENIPHE. Repéré à http://ceniphe.free. fr/revue2_files/Y.\%20mayzaud,\%20Historique\%20et\%20enjeu\%20de\%20la\%20notion\%20d'Erlebnis. pdf

Ministère de l'Éducation nationale. (2008). Organisation de l'enseignement de l'histoire des arts (école, collège, lycée). Bulletin Officiel n’32 du 28 août. Encart. Repéré à http://cache.media.education.gouv.fr/ file/32/09/0/encart_33090.pdf

Ministère de l'Éducation nationale. (2015). Parcours d'éducation artistique et culturelle. Bulletin Officiel n² 28 du 8juillet. Repéré à http://www.education.gouv.fr/pid25535/bulletin_officiel.html?cid_bo=91164

Merlin-Kajman, H. (2016). Lire dans la gueule du loup. Essai sur une zone à défendre, la littérature. Paris : Gallimard.

Mitterand, H. (1992). Les obsédés de l'objectif. L'enseignement du français en débat. Le Débat, (71), 164172.

Morizot, J. et Pouivet, R. (dir.). (2007). Dictionnaire d'esthétique et de philosophie de l'art. Paris : Armand Colin.

Parsons, M. (2010). Interpreting art through metaphors. International Journal of Art \& Design Education, 29(3), 228-235. https://doi.org/10.1111/j.1476-8070.2010.01621.x

Peirce, C. S. (1978). Écrits sur le signe. Paris : Le Seuil.

Petit, M. (2002). Éloge de la lecture : la construction de soi. Paris : Belin.

Picard, R. (1965). Nouvelle critique ou nouvelle imposture. Paris : Pauvert.

Pouivet, R. (2010). L'ontologie de l'œuvre d'art (2édition revue et augmentée). Paris : Vrin.

Privat, J.-M. (1995). Socio-logiques des didactiques de la lecture. Dans J.-L. Chiss (dir.), Didactique du français : état d'une discipline (1 édition, p. 133153). Paris : Nathan.

Réhault, S. (2013). La beauté des choses: esthétique, métaphysique et éthique. Rennes : Presses universitaires de Rennes.

Schaeffer, J.-M. (2015). L'expérience esthétique. Paris : Gallimard.

Sobczak, I. (2016). Dossier pédagogique «L'art et la matière. Galerie de sculptures à toucher». Montpellier : Musée Fabre. Repéré à http://museefabre.montpellier3m.fr/content/download/11662/88994/file/Art_et_ Matiere_NOTICES_oeuvres_et_LEXIQUE.pdf

Todorov, T. (2007). La littérature en péril. Paris : Flammarion. 
Vaugeois, D. (dir.). (2005). L'écrit sur l'art: un genre littéraire? Pau : Publications de l’Université de Pau. Vernay, J.-F. (2013). Plaidoyer pour un renouveau de l'émotion en littérature. Paris : Éditions Complicités. Vouilloux, B. (1997). Langages de l'art et relations transesthétiques. Paris/Tel Aviv : Éditions de l'Éclat.

Vouilloux, B. (2005). Les écrits sur l'art forment-ils un genre? Dans D. Vaugeois (dir.), L'écrit sur l'art: un genre littéraire (p. 3352). Pau : Publications de l'Université de Pau.

Wittgenstein, L. (1953). Recherches philosophiques (traduction française de 2005). Paris : Gallimard. 